\title{
Electronic Properties of Lithiated SnO-based Anode Materials
}

\author{
Dominik Bauer*, Teutë Bunjaku, Andreas Pedersen, and Mathieu Luisier \\ Integrated Systems Laboratory, Department of Electrical Engineering and Information Technology, \\ ETH Zürich, Gloriastrasse 35, 8092 Zürich, Switzerland
}

\begin{abstract}
In this paper we use an ab-initio quantum transport approach to study the electron current flowing through lithiated $\mathrm{SnO}$ anodes for potential applications in Li-ion batteries. By investigating a set of lithiated structures with varying lithium concentrations, it is revealed that $\mathrm{Li}_{\mathrm{x}} \mathrm{SnO}$ can be a good conductor, with values comparable to bulk $\beta$-Sn and $\mathrm{Li}$. A deeper insight into the current distribution indicates that electrons preferably follow specific trajectories, which offer superior conducting properties than others. These channels have been identified and it is shown here how they can enhance or deteriorate the current flow in lithiated anode materials.
\end{abstract}

*dobauer@iis.ee.ethz.ch

\section{INTRODUCTION}

In our modern society the increasing usage of portable electronic devices such as smartphones, tablets, or laptops, calls for the development of enhanced rechargeable batteries. Similarly, the complete replacement of conventional cars based on fossil fuel by electrical vehicles will only become possible if the storage capacity of their battery significantly improves [1, 2]. Li-ion batteries have emerged as one of the most suitable candidates in all these applications due to their relatively large energy and power densities. Still, lots of progresses remain to be done before achieving the performance limit of the Li-ion battery technology. For example, graphite, which is the most commonly used anode material with its specific capacity of $372 \mathrm{mAh} / \mathrm{g}$, is outperformed by many other components in terms of Li-storage [3 6]. This is the case of tin-based structures, whose storage capacity can reach values up to $940 \mathrm{mAh} / \mathrm{g}$ [7/9]. However, this feature comes with large volume expansions when inserting lithium ions into $\mathrm{SnO}$ or $\mathrm{SnO}_{2}$ [1, 10, 11. There are different approaches to overcome the possible performance degradation. One is the formation of a composite material by combining the host material with carbon 12 14. Another consists in arranging the active anode material into nanostructures 15-17. For the latter, it is not only essential to understand how nanosizing affects the morphology of the anode, but also how efficiently the freed or captured electrons can flow through the formed atomic systems. This work focuses on the characterization of electrical transport through nanostructured lithiated $\mathrm{SnO}$.

Experimentally investigating the different lithiation levels of $\mathrm{SnO}$ can be very time-consuming. A convenient alternative consists in using ab-initio (from first-principles) simulations, where there is no need to actually fabricate material samples. Such approaches, based on fundamental physics, are now well-established due to the increase in available computer power and the rapid improvements in computational methods [18.

The structural variations of a hypothetical lithiated $\mathrm{SnO}$ set has already been studied at the ab-initio level in Ref. [19] and agrees well with experimental data in terms of volume expansion [20]. Based on these simulations, it has been proposed that the lithiation and delithiation of $\mathrm{SnO}$ is reversible between 2 and 6.5 lithium ions per $\mathrm{SnO}$ unit, which organize themselves as Li layers. Starting from the suggested irreversible $\mathrm{Li}_{2} \mathrm{SnO}$ crystal and continuously increasing the Li concentration results in two phase transformations, one where the $\mathrm{Sn}$ atoms get dissociated from the formed $\mathrm{Li}_{2} \mathrm{O}$ matrix and another where $\mathrm{Li}$ ions are placed between $\mathrm{Sn}$ atoms and cause a large volume expansion.

This paper therefore concentrates on another aspect, the electronic properties of the anode material, which might be equally important as the structural changes. The electron conductance is indeed a critical factor that determines the efficiency of the battery charge and discharge processes. Ideally, the electrical current, which is a measure of the conductance, should reach magnitudes comparable to those of bulk $\beta$-Sn and Li. Although the presented results are specific to the lithiated $\mathrm{SnO}$ system, the proposed simulation methodology can be applied to any electrode material and shed light on the interplay between morphological and electronic transport effects.

The paper is organized as follows. In Section II] the full simulation approach is described and explained. Section III discusses the electron current flowing through the $\mathrm{Li}_{\mathrm{x}} \mathrm{SnO}$ structures $(2 \leq \mathrm{x} \leq 6.5)$. The total current will be analyzed first before insight into the preferred electron trajectories is provided. Afterwards, a Bader charge analysis [21] will be used to investigate the carrier distribution and derive its influence on the current values. The last subsection focuses on the fully lithiated $\mathrm{Li}_{6.5} \mathrm{SnO}$ configuration and its peculiar characteristics.

\section{SIMULATION APPROACH}

To model the electronic properties of lithiated $\mathrm{SnO}$ anodes an approach relying on density-functional theory 


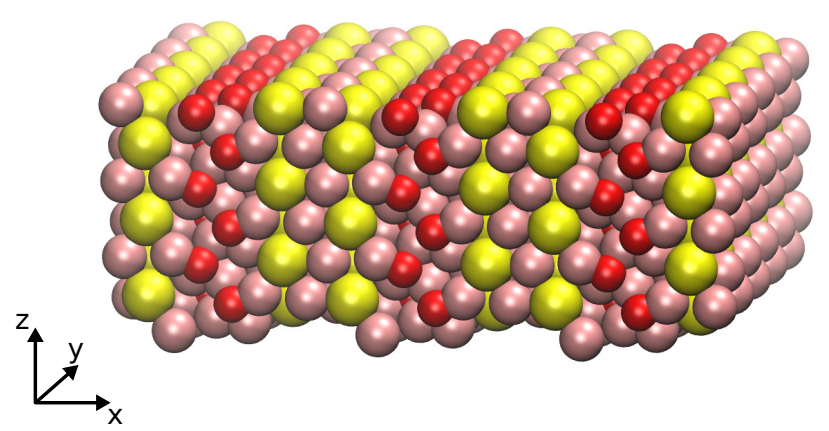

Figure 1. Atomic structure of $\mathrm{Li}_{4} \mathrm{SnO}$ with four lithium ions per SnO host unit. The pink, red, and yellow spheres correspond to lithium, oxygen, and tin atoms, respectively.

(DFT) is used. It is based on the plane-wave pseudopotential method, as implemented in the VASP code 22, 23, within the generalized gradient approximation [24], as well as the projector-augmented wave formalism [25]. The number of k-points discretizing the Brillouin zone of each atomic structure is chosen to be at least $3 \times 11 \times 11$. The plane-wave kinetic energy cutoff is set to $600 \mathrm{eV}$ with an electronic convergence criteria of $10^{-6}$. The VASP calculations are performed for the smallest possible rectangular unit cell that represents the considered system, i.e. 4 atoms of tin and oxygen in the smallest case and 16 in the largest. The produced BlochHamiltonian matrix corresponding to the chosen periodic crystal is transformed into a set of maximally localized Wannier functions (MLWFs) with the Wannier90 tool [26]. This step is needed for transport calculations, as only a sparse Hamiltonian matrix allows for the injection of electrons into a non-periodic simulation domain. The accuracy of the MLWF transformation has been validated by comparing the bandstructure obtained with VASP to that resulting from Wannier90. As an illustration, Figure 2 shows the bandstructure of bulk Li. By keeping four Wannier functions per Li atom (one with an s-like, three with a p-like symmetry), it can be observed that around the Fermi level, both calculation methods agree very well. Larger discrepancies are visible at higher energies, but they can be ignored since those states do not contribute to the transport properties.

The ballistic conductance of the $\mathrm{Li}_{\mathrm{x}} \mathrm{SnO}$ anodes can then be calculated in two ways. In the first one, the LandauerBüttiker formula [27] is recalled to evaluate the total current flowing through the anode material

$$
I_{\text {total }}=\frac{2 e}{\hbar} \sum_{k} \int \frac{d E}{2 \pi} T(E, k)\left[f\left(E, E_{F L}\right)-f\left(E, E_{F R}\right)\right]
$$

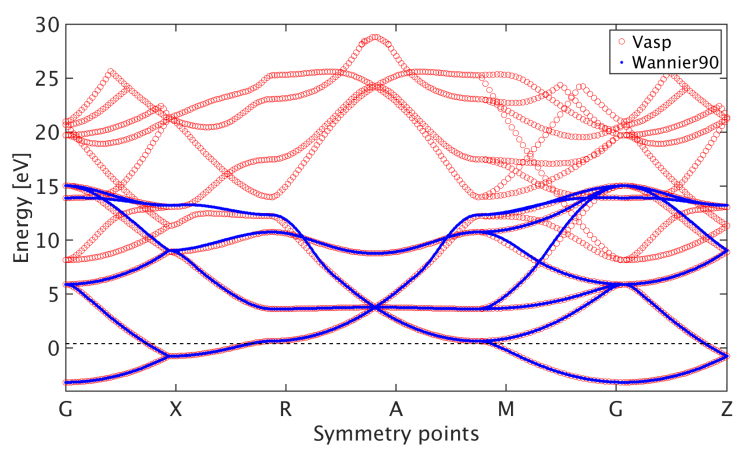

Figure 2. Bandstructure of bulk Li at high symmetry points in the Brillouin zone. The red curves refer to results obtained with VASP [22, 23], the blue curves with Wannier90 [26]. The dashed black line indicates the Fermi energy.

where $e$ is the elementary charge, $\hbar$ the reduced Planck constant, $T(E, k)$ the energy- and momentum-dependent transmission function through the anode, $f\left(E, E_{F L / R}\right)$ the Fermi-Dirac distribution function of electrons in the added left $(\mathrm{L})$ and right $(\mathrm{R})$ contact regions, and the factor 2 accounts for the spin degeneracy [28]. In Eq. (1), a small bias difference $\triangle V \leq 1 \cdot 10^{-3} \mathrm{~V}$ is assumed to be applied between the left and right end of the $\mathrm{Li}_{\mathrm{x}} \mathrm{SnO}$ system. This value is appropriate for the short length of the studied part of a potential full anode. To compute $T(E, k)$ at a given energy $E$ and momentum $k$, the bandstructure of the primitive rectangular unit cell can be employed. The latter can be rapidly determined in the entire Brillouin zone with the generated MLWF Hamiltonian. Each time a band with a positive velocity crosses the selected $(E, k)$ pair, $T(E, k)$ increases by one unit, i.e. if there are $n$ bands fulfilling the required criterion, then $T(E, k)=n$. While this approach might be computational very efficient, it does not offer any insight into the trajectories that electrons follow through $\mathrm{Li}_{\mathrm{x}} \mathrm{SnO}$. Only the current value is known.

To go one step further and identify the preferred current paths, a ballistic quantum transport (QT) solver can be utilized. For that purpose, the MLWF-Hamiltonian $\mathrm{H}_{\mathrm{MLWF}}$ of the primitive rectangular unit cell is scaled up to enable the simulation of larger structures. The resulting supercell matrix exhibits a block tri-diagonal shape. Here, it is typically composed of four primitive cells along the transport direction [29].

The QT calculations are performed within the wave function (WF) or Non-equilibrium Green's function (NEGF) formalism after importing the externally created MLWF Hamiltonian matrix [30]. In NEGF, the current between atoms $i$ and $j$ is given by

$$
I_{d, i j}=\frac{2 e}{\hbar} \sum_{k, \sigma_{1}, \sigma_{2}} \int \frac{d E}{2 \pi}\left(H_{M L W F_{i j}}^{\sigma_{1} \sigma_{2}}(k) \cdot G_{j i}^{<\sigma_{2} \sigma_{1}}(E, k)\right.
$$




$$
\left.-G_{i j}^{<\sigma_{1} \sigma_{2}} \cdot H_{M L W F_{j i}}^{\sigma_{2} \sigma_{1}}(k)\right)
$$

In Eq. (2), $H_{M L W F_{i j}}^{\sigma_{1} \sigma_{2}}(k)$ is the momentum-dependent Hamiltonıan matrix element between atoms $i$ and $j$ and between the Wannier functions $\sigma_{1}$ and $\sigma_{2}$, while $G_{i j}^{<\sigma_{1} \sigma_{2}}(E, k)$ is the lesser Green's function coupling atoms $i$ and $j$ as well as Wannier functions $\sigma_{1}$ and $\sigma_{2}$ at energy $E$ and momentum $k$. Note that because large supercells are constructed for transport, the size of the Brillouin zone drastically decreases and no summation over momentum is needed any more. The numerical problem reduces to a $\Gamma$-point calculation. The NEGF approach enables not only to extract the local current between different orbitals, but also includes the calculation of the total current via Eq. (1) by indirectly delivering the transmission function $T(E, k)$. Both of the above mentioned methods have been applied to our set of structures. However, to consistently compare the total current values to the current per bond analysis later on, the computation in this paper is restricted to the quantum transport approach.

\section{RESULTS}

\section{A. Total current}

As a first step the total current flowing through the $\mathrm{Li}_{\mathrm{x}} \mathrm{SnO}$ structures is determined with Eq. (1). Figure 4 reports the simulation results, both along the $\mathrm{y}$ - and $\mathrm{z}$ axis, as defined in Fig. 1. The current along the x-axis is omitted because the periodically repeated oxide layers along this direction prevents any electron transport. Such structure can be clearly seen in Fig. 1 for $\mathrm{Li}_{4} \mathrm{SnO}$, but is a common feature of all $\mathrm{Li}_{\mathrm{x}} \mathrm{SnO}$ structures with $2 \leq \mathrm{x} \leq 6.5 \mathrm{Li}$ layers. To further support this statement, the bandstructures of all atomic systems have been investigated. It has been found that all structures are metallic, except along the $\mathrm{k}_{\mathrm{x}}$-axis $(\mathrm{R}$ - A line in the Brillouin zone) where the bands have a flat dispersion and are separated by a large band gap, thus hindering electron transport along the $\mathrm{x}$-axis and leaving this direction insulating. Fig. 3illustrates this behavior with two representative bandstructures, the one of $\mathrm{Li}_{4} \mathrm{SnO}$ and $\mathrm{Li}_{6} \mathrm{SnO}$. Orbital projected bands have also been generated as an additional information, indicating that Sn d orbitals are contributing the most to the bands around the Fermi level. When $T(E, k)$ is evaluated with NEGF in Eq. (1), the computational burden becomes very high. It can be reduced by decreasing the size of the supercell and the number of energy points, which introduces a small error in the current of maximum $0.4 \cdot 10^{11} \mathrm{~A} / \mathrm{m}^{2}$.

The total current for bulk $\beta$-Sn and bulk Li has also been calculated and compared to lithiated $\mathrm{SnO}$. The values are $7.3 \cdot 10^{11} \mathrm{~A} / \mathrm{m}^{2}$ and $7.4 \cdot 10^{11} \mathrm{~A} / \mathrm{m}^{2}$, respectively. It can (a)

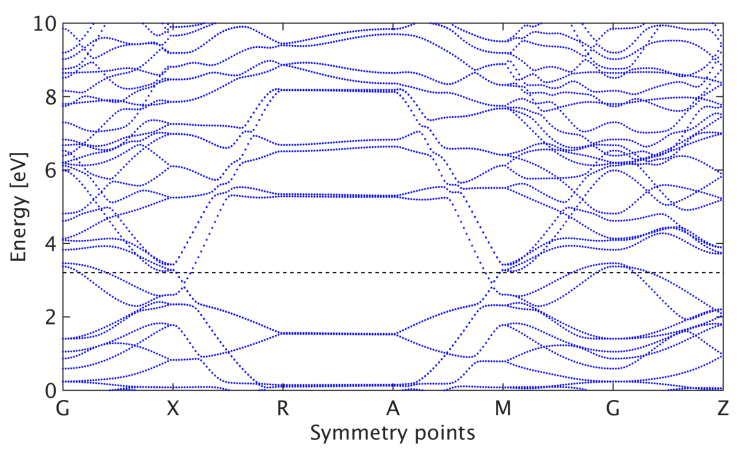

(b)

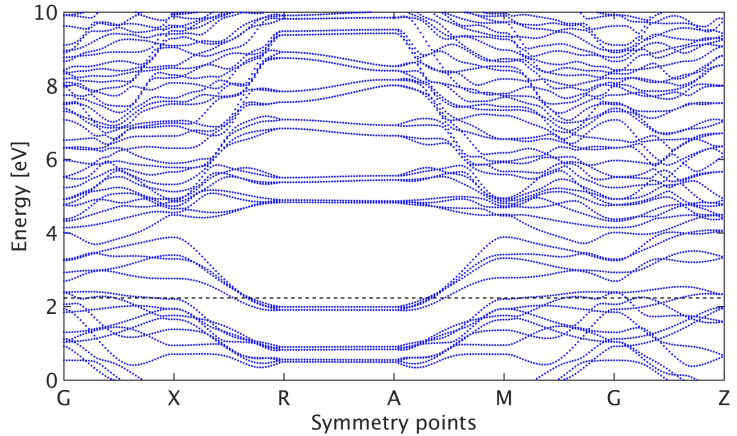

Figure 3. Bandstructure of $\mathrm{Li}_{4} \mathrm{SnO}$ (a) and $\mathrm{Li}_{6} \mathrm{SnO}$ (b) at high symmetry points in the Brillouin zone. The bands between $\mathrm{R}$ and $\mathrm{A}$ correspond to transport along the $\mathrm{x}$-axis. The dashed line indicates the Fermi level of the respective structure.

therefore be concluded that $\mathrm{Li}_{\mathrm{x}} \mathrm{SnO}$ as an anode material exhibits good electronic properties, up to $60 \%$ of those of bulk $\beta$-Sn and bulk Li. Consequently, they are not expected to limit the charge and discharge processes of a battery system with perfectly ordered electrodes. The situation might change after few cycles, when different grained lithiation levels start to be mixed together. The influence of disorder is however out-of-the-scope of this paper.

It can be seen that the current in Fig. 4 does not follow a monotonic behavior with respect to the amount of inserted lithium. At first, when going from 2 to $3 \mathrm{Li}$ ions per $\mathrm{SnO}$ unit, there is a large increase in current. According to Ref. [19] this corresponds to the first phase transformation of the anode. When inserting the first reversible $\mathrm{Li}$ layer into $\mathrm{Li}_{2} \mathrm{SnO}$, the oxygen region becomes fully filled up with lithium and the observed Sn bilayer is transformed into a separate monolayer whose distance to the $\mathrm{O}$ atoms is higher than in $\mathrm{Li}_{2} \mathrm{SnO}$. This separated Sn monolayer offers highly conductive paths, leading to an increase in total current.

The second phase transformation occurs when a second reversible Li layer is inserted, so transitioning from $\mathrm{Li}_{3} \mathrm{SnO}$ to $\mathrm{Li}_{4} \mathrm{SnO}$. The anode volume starts expanding in the direction perpendicular to the oxygen plane, as lithium is now placed in between the Sn atoms. This relocation introduces a larger distance between neighbor- 


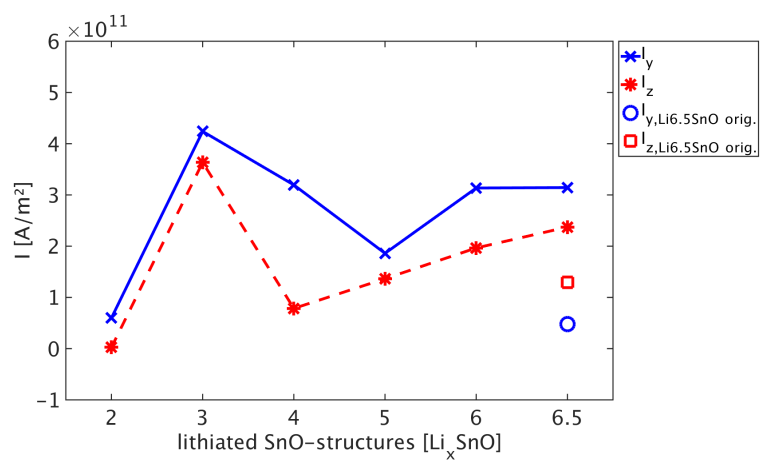

Figure 4. Total current per area for the studied $\mathrm{Li}_{\mathrm{x}} \mathrm{SnO}$ structure measured from one contact to the other along the $\mathrm{y}$ and z-directions (see Fig. 1p, $I_{y}$ and $I_{z}$, respectively. The $\mathrm{x}$-direction is insulating because of the dense oxide layer separating conductive segments. The horizontal axis shows the amount of lithium ions per $\mathrm{SnO}$ host unit. For 6.5 layers, the two sets of markers (cross/asterisk and circle/square) refer to the total current value obtained from different configurations, as discussed in the last subsection of the paper.

ing Sn atoms and causes a decrease of the total current. Along the y-direction, the effect is less pronounced because of the presence of zig-zag Sn-channels that still provide a good conductance. This will be discussed later. The larger drop in $I_{z}$ can be attributed to the absence of such channels in the z-direction. When inserting the fifth Li layer into $\mathrm{SnO}, I_{y}$ keeps decreasing as more $\mathrm{Li}$ atoms relocate between the Sn atoms, thus finally dissolving the Sn-channels. In the other direction (z), the current magnitude reincreases because lithium channels start to form. However, as the conductance per lithium atom is lower than in the Sn-channels, the growth rate of the current is not comparable to the $\mathrm{Li}_{2} \mathrm{SnO}-\mathrm{Li}_{3} \mathrm{SnO}$ transition. This finding might seem counterintuitive since the current of both bulk $\beta$-Sn and bulk Li lie in the same range so that Sn- and Li-based channels should offer similar conductance values. The reason behind this surprising behavior will be addressed later on.

At the next lithiation levels, the current steadily increases, as more $\mathrm{Li}$ atoms are available for transport. Note that the current values depicted at $6.5 \mathrm{Li}$ layers in Fig. 4 correspond to different $\mathrm{Li}_{6.5} \mathrm{SnO}$ configurations. Additional knowledge about bond-resolved current trajectories are needed to explain them. Until then, the focus is set on the rest of the structures.

\section{B. Current per bond}

With the help of Eq. (2) the current between two specific atoms can be evaluated by determining all incoming and outgoing contributions from their neighbors. After summing up those values the current components from Sn-to-Sn, Li-to-Li, Sn-to-Li, and Li-to-Sn can be visu- alized for the y- and z-direction, as reported in Fig. 5 . Both plots show similar results: following the first phase transformation where $\mathrm{Li}_{2} \mathrm{SnO}$ evolves into $\mathrm{Li}_{3} \mathrm{SnO}$, the Sn atoms form separate channels, which is confirmed by the increased Sn-to-Sn current. After this initial boost, the Sn-Sn current decreases because the insertion of additional $\mathrm{Li}$ ions pushes the $\mathrm{Sn}$ atoms away from each other, thus negatively affecting the Sn channels. This trend continues as the lithium concentration increases. At $\mathrm{Li}_{6} \mathrm{SnO}$, the distance between neighboring $\mathrm{Sn}$ atoms is so large that the Sn-to-Sn current contribution becomes negligible. Note that a negative current is equivalent to reflections due to the absence of connections along the transport direction. At the same time, the $\mathrm{I}_{\mathrm{Li}-\mathrm{Li}}$ component slowly increases due to the presence of more and more Li ions in the anode and shorter distances between them. Thereby, the conductance through $\mathrm{Li}$ atoms steadily substitutes that of Sn by forming alternative channels. Hence, two opposite trends compete with each other, the decrease of the Sn-to-Sn contributions and the increase of the Li-to-Li ones. Since the latter dominates, the total current increases between $\mathrm{Li}_{5} \mathrm{SnO}$ and $\mathrm{Li}_{6} \mathrm{SnO}$.

Important information about the current flow resides in the $\mathrm{I}_{\mathrm{Sn}-\mathrm{Li}} / \mathrm{I}_{\mathrm{Li}-\mathrm{Sn}}$ component. Figure 5 reveals that it has a high influence throughout the whole lithiation process. Hence, even though direct connections between Sn atoms vanish, those particles still significantly contribute to the overall current flow, but indirectly via detours through Li atoms.

In the chosen MLWF approach one atom is connected to several neighbors, but not all of them contribute equally to the current magnitude. By only keeping the connections with the highest weight it is possible to draw a trajectory map of the current. This is done by representing the largest current components through a directional arrow that starts from the atom position and points to the dominant direction. An example is given in Fig. 6 for $\mathrm{Li}_{4} \mathrm{SnO}$. It should be noted that the current along the $\mathrm{y}$-direction is significantly higher than along the z-axis. This is due to the zig-zag alignment of the tin atoms along $\mathrm{y}$, which resembles the configuration of bulk $\beta$-Sn and is therefore favorable to electron transport. Along $\mathrm{z}$, the current flow is perpendicular to these zig-zag channels so that the distance between two Sn atoms situated in adjacent channels is too large to allow for a direct connection. Thus, neighboring Li atoms must be used as surrogate links between Sn channels. Since this process is not as efficient as direct Sn-Sn coupling a decrease in total current is observed, which explains the different behaviors along the $y$ - and z-direction mentioned in the previous section. 
(a)

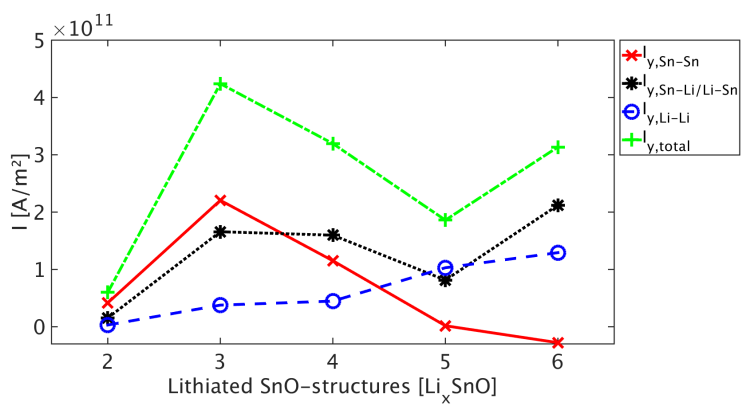

(b)

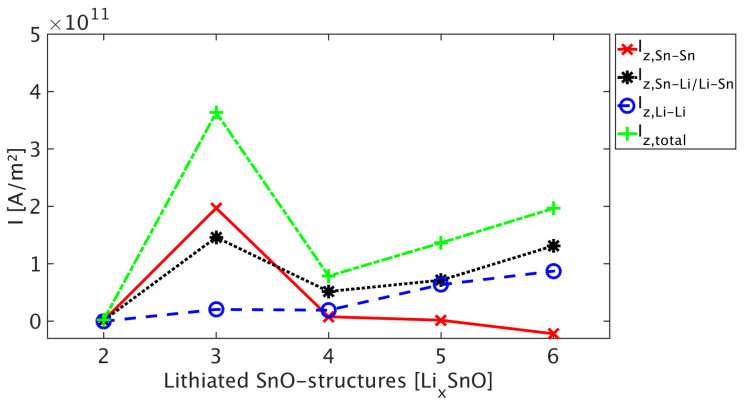

Figure 5. Current components per area for each lithiated structure between $\mathrm{Li}_{2} \mathrm{SnO}$ and $\mathrm{Li}_{6} \mathrm{SnO}$ along the $\mathrm{y}-(\mathrm{a})$ and z- (b) direction. The same plotting conventions as in Fig. 4 are used. The red solid, black dotted, and blue dashed curves show the current between $\mathrm{Sn}-\mathrm{Sn}, \mathrm{Sn}-\mathrm{Li} / \mathrm{Li}-\mathrm{Sn}$, and Li$\mathrm{Li}$ atoms, respectively. The green dash-dotted curve indicates the total current.

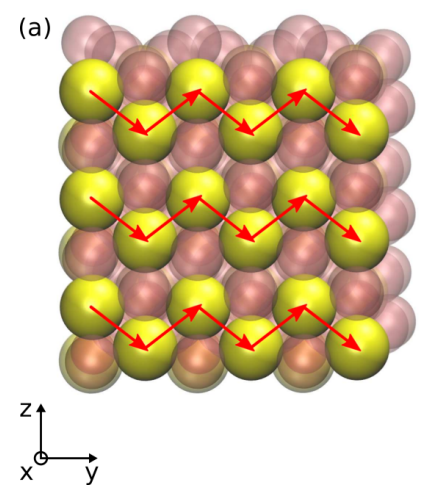

(b)

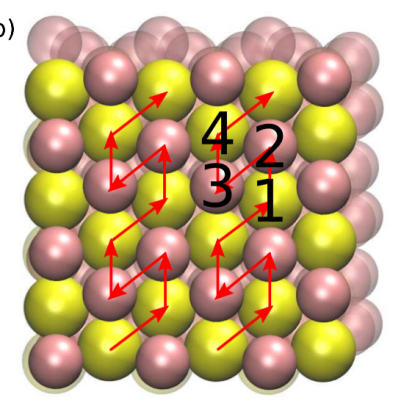

Figure 6. Current map for $\mathrm{Li}_{4} \mathrm{SnO}$ along the $\mathrm{y}$-(a) and z-(b) direction. The dominant current flow is represented by the red arrows. In (b), the current follows a non-direct path from atoms 1 to 4 , always hopping to the closest neighbor, i.e. the distance between atoms 1 and 2 is shorter than between 1 and 3. Similarly, atom 2 is closer to atom 3 rather than 4 .

\section{Charge distribution}

Our simulations indicate that the electrical current flowing through chains of $\mathrm{Li}$ atoms is considerably lower than through Sn channels, whereas bulk Li and bulk $\beta$-Sn exhibit comparable current values. To determine why Li

\begin{tabular}{|c|c|c|c|}
\hline & $\mathrm{Sn}[\mathrm{e}]$ & $\mathrm{O}[\mathrm{e}]$ & $\mathrm{Li}[\mathrm{e}]$ \\
\hline \hline $\mathrm{Li}_{2} \mathrm{SnO}$ & 4.15 & 7.50 & 0.16 \\
\hline $\mathrm{Li}_{3} \mathrm{SnO}$ & 4.80 & 7.70 & $(0.17-0.20)$ \\
\hline $\mathrm{Li}_{4} \mathrm{SnO}$ & 5.60 & 7.70 & $(0.16-0.19)$ \\
\hline $\mathrm{Li}_{5} \mathrm{SnO}$ & $(5.60-7.20)$ & 7.70 & $(0.16-0.19)$ \\
\hline $\mathrm{Li}_{6} \mathrm{SnO}$ & 7.20 & 7.70 & $(0.17-0.20)$ \\
\hline $\mathrm{Li}_{6.5} \mathrm{SnO}$ orig. & $(7.50-7.60)$ & 7.70 & $(0.17-0.20)$ \\
\hline $\mathrm{Li}_{6.5} \mathrm{SnO}$ adj. & $(7.20-7.80)$ & 7.70 & $(0.17-0.23)$ \\
\hline bulk $\mathrm{Li}$ & 0 & 0 & 1 \\
\hline bulk $\beta$-Sn & 4 & 0 & 0 \\
\hline
\end{tabular}

Table I. Electron charge distribution in each of the considered $\mathrm{Li}_{\mathrm{x}} \mathrm{SnO}$ structures as well as in bulk $\mathrm{Li}$ and bulk $\beta$-Sn. Two $\mathrm{Li}_{6.5} \mathrm{SnO}$ configurations, one labeled original (orig.), the other adjusted (adj.) structure, are presented. The difference between them will be discussed in Section IIID

and Sn atoms behave differently when they are mixed together in $\mathrm{Li}_{\mathrm{x}} \mathrm{SnO}$ structures, a Bader charge analysis can be utilized [21]. It provides an accurate estimation of the charge distributed on each atom based on the VASP outputs. Table $\mathbb{I}$ summarizes the results for each of the $\mathrm{Li}_{\mathrm{x}} \mathrm{SnO}$ structure, as well as for bulk $\mathrm{Li}$ and bulk $\beta$-Sn.

When comparing the bulk to the $\mathrm{Li}_{\mathrm{x}} \mathrm{SnO}$ structures, it appears that in the latter case, the charge distribution strongly influences the electronic transport properties. The highly reactive $\mathrm{Li}$ ions, on average, give away more than $80 \%$ of their charge to the $\mathrm{Sn}$ and $\mathrm{O}$ atoms. In the $\mathrm{Li}_{2} \mathrm{SnO}$ case, the two Li layers are tightly bound to the oxygen atoms, to which they both transfers 0.75 electron to form an irreversible $\mathrm{Li}_{2} \mathrm{O}$ layer. The $\mathrm{Li}$ ions that are subsequently added to $\mathrm{Li}_{2} \mathrm{SnO}$ donate roughly 0.8 electron to their neighboring $\mathrm{Sn}$ atoms. As a consequence of these charge transfer processes, the conductance of the $\mathrm{Li}$ ions decreases.

The correlation between the charge and conductance variations is not obvious at all and requires therefore a careful inspection. This is done by investigating a simple composite SnLi system. A primitive unit cell of bulk $\beta$-Sn with four atoms is considered. A Bader charge analysis is performed and the current per bond is simulated after replacing the $\mathrm{Sn}$ atoms one by one with $\mathrm{Li}$ atoms. The relaxed configurations have only negligible bond connections between dissimilar atom types along the z-direction. In this way, pure lithium and tin atomic channels can be created and their current compared to each other. The results are presented in Figure 7. In subplot (a), the inserted $\mathrm{Li}$ donates 0.8 electron to the two adjacent $\mathrm{Sn}$ atoms, whose charge becomes 4.4 electrons each. The last $\mathrm{Sn}$ atom keeps its original charge (4 electrons).

In this configuration, the current per bond that flows through the pure $\mathrm{Li}$ channel along the $\mathrm{z}$-axis is equal to $1.4 \cdot 10^{10} \mathrm{~A} / \mathrm{m}^{2}$ and is much smaller than for the Sn-Sn cases $\left(4.8 \cdot 10^{10} \mathrm{~A} / \mathrm{m}^{2}\right.$ for the atoms with 4.4 electrons, $4.0 \cdot 10^{10} \mathrm{~A} / \mathrm{m}^{2}$ for those with 4.0 electrons). This is a first indication that the charge transfer to neighboring 


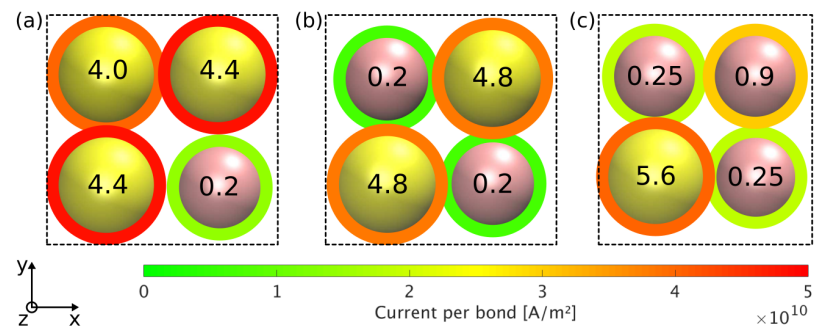

Figure 7. $\beta$-Sn structures where one (a), two (b), and three (c) tin atoms have been replaced by a $\mathrm{Li}$ atom. The numbers on the atoms show the calculated electron charge. The colored circles around the atoms refer to the computed current magnitude per bond along the $\mathrm{z}$-direction. The dashed rectangle represent the primitive unit cell. All of the displayed atoms lie in the same z-plane.

Sn atoms negatively affects the Li conductance, while it benefits to the Sn channels whose current increases.

This trend is confirmed in Fig. 7(b) where again the $\mathrm{Li}$ atoms give away 0.8 electron to the Sn elements sitting in their immediate vicinity and thus exhibit much lower currents $\left(0.7 \cdot 10^{10} \mathrm{~A} / \mathrm{m}^{2}\right.$ for the Li-Li channels vs. $3.8 \cdot 10^{10} \mathrm{~A} / \mathrm{m}^{2}$ for the Sn-Sn ones). Finally, the same observation can be made in Fig. 7(c). The Li atoms that have been added all donate a fraction of their charge to the Sn atom they surround, but not in the same proportion. Essentially, the particle which lost only $10 \%$ of its charge carries a higher current $\left(3.0 \cdot 10^{10} \mathrm{~A} / \mathrm{m}^{2}\right)$ than the ones which gave away 0.8 electron $\left(I_{d}=1.8 \cdot 10^{10} \mathrm{~A} / \mathrm{m}^{2}\right)$. Furthermore, these current magnitudes remain much lower than in the $\mathrm{Sn}-\mathrm{Sn}$ channels $\left(4.0 \cdot 10^{10} \mathrm{~A} / \mathrm{m}^{2}\right)$. From these three numerical experiments detailed in Fig. 7 it can be definitively concluded that when Li atoms sit close to Sn ones, a transfer of charge takes place between both atom types. Thereby, the charge loss leads to a current reduction in the Li-Li channels.

\section{D. $\mathrm{Li}_{6.5} \mathrm{SnO}$ : original structure vs. adjusted configuration}

When simulating the original $\mathrm{Li}_{6.5} \mathrm{SnO}$ structure found in Ref. [19, it has been discovered that the total current suddenly drops, as compared to $\mathrm{Li}_{6} \mathrm{SnO}$. This unexpected behavior is shown in Fig. 4. The original $\mathrm{Li}_{6.5} \mathrm{SnO}$ configuration relaxes in such a way that no alternating Sn-Li-Sn channels are formed, as illustrated in Fig. 8(a). However, by studying the lower lithiated arrangements and applying the lessons learnt in these cases, an "adjusted" structure with more favorable current paths can be created and relaxed according to the prescriptions from Ref. [19]. Its total energy is $0.18 \%$ higher than in the original structure with a similar charge distribution, as can be seen in Table [. Altogether, this makes it a possible alternative

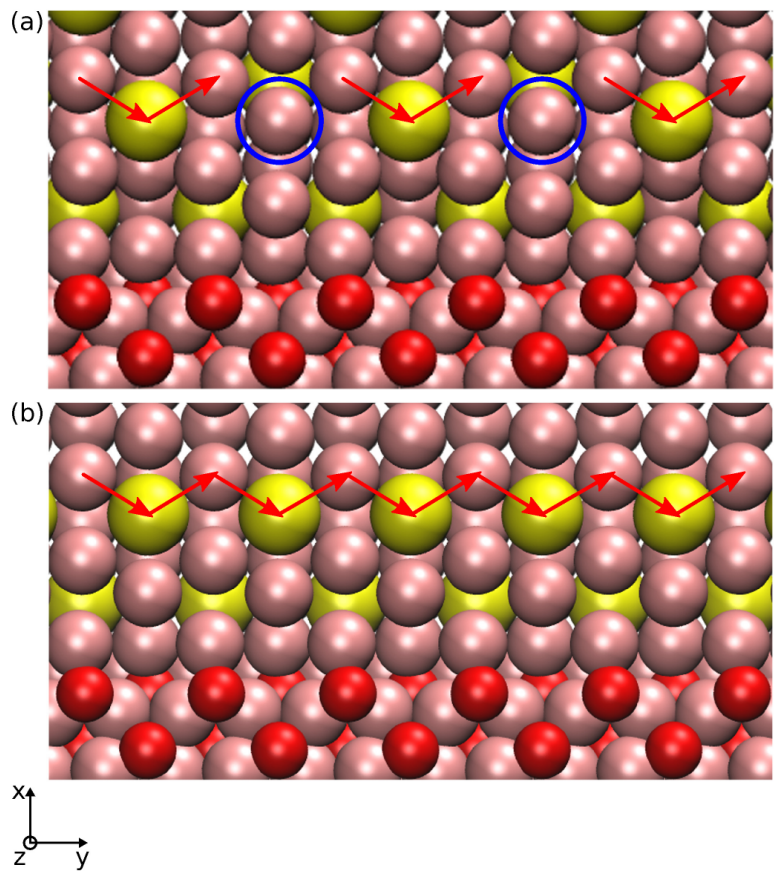

Figure 8. Atomic configuration and dominant current path for the original (a) and the adjusted (b) configuration of $\mathrm{Li}_{6.5} \mathrm{SnO}$. In (a), the blue circles refer to lithium spots, which are replaced by $\mathrm{Sn}$ atoms in the adjusted configuration to form alternating Sn-Li-Sn channels.

configuration.

Through transport simulations the dominant current trajectories can be again identified. In Figure 8 , the focus is set on the differences between the original and the adjusted structure. In subplot (a), it can be observed that the missing Sn atoms at the circle locations do not allow for the completion of the Sn-Li-Sn channels that carry most of the current in the $\mathrm{Li}_{5} \mathrm{SnO}$ or $\mathrm{Li}_{6} \mathrm{SnO}$ structures. With the atomic rearrangement in Fig. 8(b), these channels reappear, which increases the total current value. Hence, the trend that started with the lesser lithiated $\mathrm{SnO}$ materials continues, as depicted with the cross/asterisk markers in Fig. 4. The unfavorable current flow in the original structure could come from the fact that the lithium load has already reached its theoretically predicted limit of $\mathrm{Li}_{4.4} \mathrm{Sn}\left(+\mathrm{Li}_{2} \mathrm{O}\right)$ 31]. It has been verified that forcing a similar atomic arrangement as in Fig. 8(a) for lower lithiated structures, e.g. $\mathrm{Li}_{6} \mathrm{SnO}$ or $\mathrm{Li}_{5} \mathrm{SnO}$, does not reduce their total energy.

\section{CONCLUSION}

We have investigated the electronic properties of perfectly ordered lithiated $\mathrm{SnO}$ at different lithium concentrations with the help of density-functional theory and 
quantum transport simulations. This work has mainly shed light on the influence of the atomic arrangements on the total electrical current by evaluating the flow along each bond connecting two atoms as well as by determining the charge distribution in composite systems. The interplay between pure Sn, pure Li, and mixed Sn-Li channels has been analyzed to explain the current behavior as a function of the Li level. All in all, it has been found that favorable current paths exist in $\mathrm{Li}_{\mathrm{x}} \mathrm{SnO}$ with conductance values close to what can be obtained in bulk $\beta$-Sn and bulk Li, except in the direction orthogonal to the $\mathrm{Li}_{2} \mathrm{O}$ layers that are created in the first irreversible lithiation step. This confirms that $\mathrm{SnO}$ is a promising anode material, provided that the significant volume changes it undergoes can be well managed. More generally, the proposed approach to study electronic transport in battery electrodes can now be used to explore other atomic systems.

\section{ACKNOWLEDGMENTS}

This research was supported by the European Research Council under Grant Agreement No 335684-E-MOBILE.

[1] M. Liang and L. Zhi, Journal of Materials Chemistry 19, 5871 (2009).

[2] H. Wu and Y. Cui, Nano Today 7, 414 (2012).

[3] H. Li, K. Yu, H. Fu, B. Guo, X. Lei, and Z. Zhu, The Journal of Physical Chemistry C 119, 7959 (2015).

[4] M. Winter and J. O. Besenhard, Electrochimica Acta 45, 31 (1999).

[5] S.-C. Chao, Y.-C. Yen, Y.-F. Song, H.-S. Sheu, H.-C. Wu, and N.-L. Wu, Journal of The Electrochemical Society 158, A1335 (2011).

[6] P. Meduri, C. Pendyala, V. Kumar, G. U. Sumanasekera, and M. K. Sunkara, Nano letters 9, 612 (2009).

[7] Y. Idota, T. Kubota, A. Matsufuji, Y. Maekawa, and T. Miyasaka, Science 276, 1395 (1997).

[8] C.-M. Park, J.-H. Kim, H. Kim, and H.-J. Sohn, Chemical Society Reviews 39, 3115 (2010).

[9] N. Tamura, R. Ohshita, M. Fujimoto, M. Kamino, and S. Fujitani, Journal of The Electrochemical Society 150,
A679 (2003).

[10] J. Besenhard, J. Yang, and M. Winter, Journal of Power Sources 68, 87 (1997).

[11] T. Brousse, R. Retoux, U. Herterich, and D. Schleich, Journal of The Electrochemical Society 145, 1 (1998).

[12] Y. Xu, Y. Zhu, Y. Liu, and C. Wang, Advanced Energy Materials 3, 128 (2013).

[13] X. Zhou, L.-J. Wan, and Y.-G. Guo, Advanced materials 25, 2152 (2013).

[14] Y. Yu, L. Gu, C. Zhu, P. A. Van Aken, and J. Maier, Journal of the American Chemical Society 131, 15984 (2009).

[15] L. Bazin, S. Mitra, P. L. Taberna, P. Poizot, M. Gressier, M. J. Menu, A. Barnabé, P. Simon, and J.-M. Tarascon, Journal of Power Sources 188, 578 (2009).

[16] Y. Zhao, X. Li, B. Yan, D. Li, S. Lawes, and X. Sun, Journal of Power Sources 274, 869 (2015).

[17] A. R. Kamali and D. J. Fray, Rev. Adv. Mater. Sci 27, 14 (2011).

[18] A. Kohan, G. Ceder, D. Morgan, and C. G. Van de Walle, Physical Review B 61, 15019 (2000).

[19] A. Pedersen, P. A. Khomyakov, and M. Luisier, Physical Review Applied 4, 034005 (2015).

[20] M. Ebner, F. Marone, M. Stampanoni, and V. Wood, Science 342, 716 (2013).

[21] W. Tang, E. Sanville, and G. Henkelman, Journal of Physics: Condensed Matter 21, 084204 (2009).

[22] G. Kresse and J. Furthmüller, Physical review B 54, 11169 (1996).

[23] G. Kresse and J. Furthmüller, Computational Materials Science 6, 15 (1996).

[24] J. P. Perdew, K. Burke, and M. Ernzerhof, Physical review letters 77, 3865 (1996).

[25] P. E. Blöchl, Physical Review B 50, 17953 (1994).

[26] A. A. Mostofi, J. R. Yates, Y.-S. Lee, I. Souza, D. Vanderbilt, and N. Marzari, Computer physics communications 178, 685 (2008).

[27] M. Büttiker, Y. Imry, R. Landauer, and S. Pinhas, Physical Review B 31, 6207 (1985).

[28] S. Datta, Electronic transport in mesoscopic systems (Cambridge university press, 1997).

[29] Á. Szabó, Dissipative quantum transport simulations in two-dimensional semiconductor devices from first principles, Ph.D. thesis, ETH Zürich (2016), doi:10.3929/ethza-010659234.

[30] M. Luisier, A. Schenk, W. Fichtner, and G. Klimeck, Physical Review B 74, 205323 (2006).

[31] I. A. Courtney and J. Dahn, Journal of The Electrochemical Society 144, 2045 (1997). 